\title{
On-line Desalting of Physiologically Derived Fluids in Conjunction With Capillary Isoelectric Focusing-Mass Spectrometry
}

\author{
Nigel J. Clarke and Andy J. Tomlinson \\ Biomedical Mass Spectrometry Facility, Department of Biochemistry and Molecular Biology, Mayo Clinic, \\ Rochester, Minnesota, USA \\ Stephen Naylor \\ Biomedical Mass Spectrometry Facility, Department of Biochemistry and Molecular Biology, Department of \\ Pharmacology, Mayo Clinic, Rochester, Minnesota, USA
}

Capillary isoelectric focusing (cIEF) coupled to mass spectrometry (cIEF-MS) offers a potentially very powerful analytical tool for the investigation of physiological samples. The high resolving capabilities of CIEF in combination with the high sensitivity and enhanced structural information provided by MS is highly desirable for the analysis of complex samples. However a major limitation of the technique has always been the requirement to desalt samples prior to cIEF analysis. Such desalting normally occurs off-line and therefore adds complexity and the possibility of sample loss or contamination. In this study we demonstrate the use of a modified cIEF protocol which enables samples containing physiological levels of salts to be desalted on-line, within the cIEF capillary. This new technique is very fast and efficient, allowing the direct analysis of a physiologically derived fluid that contains a complex mix of proteins, such as human cerebrospinal fluid by cIEF-MS in a single step experiment. (J Am Soc Mass Spectrom 1997, 8, 743-748) (C) 1997 American Society for Mass Spectrometry

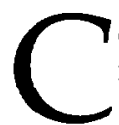
apillary isoelectric focusing (cIEF) carried out in fused silica capillaries was originally demonstrated by Hjertén and Zhu over ten years ago [1]. It has subsequently found widespread use in the separation and analysis of polypeptide and protein mixtures [2-4]. The two major advantages of clEF over conventional slab gel electrophoresis are the enhanced separation efficiencies as well as a substantial reduction in Joule heating [5]. Experimentally, the cIEF process consists of an initial focusing step that is based on the isoelectric point $(\mathrm{pI})$ values of individual analytes. It is subsequently followed by an analyte mobilization stage affected either by electrophoresis or pressure [6]. This is shown schematically in Figure 1. Capillary IEF has been used extensively with conventional UV detection; however, the use of cIEF coupled to mass spectrometry (cIEF-MS) has to date received scant attention $[7,8]$. The use of cIEF-MS affords a highly efficient separation technique with the capability to structurally characterize analytes derived from complex mixtures. This is

Address reprint requests to Stephen Naylor, Biomedical Mass Spectrometry Facility, Department of Biochemistry and Molecular Biology, Department of Pharmacology, Mayo Clinic, Rochester, MN 55905. E-mail: naylor.stephen@ mayo.edu

Initial findings were first presented at $\mathrm{HPCE}^{\prime} 97$ in Anaheim, $\mathrm{CA}$, January $26-30,1997$. potentially a very powerful combination in the analysis of unknown components derived from physiologic fluids such as urine, saliva, cerebrospinal fluid (CSF), tears, plasma, and serum. It affords the possibility of rapidly analyzing such complex mixtures and characterizing markers of disease states.

Unfortunately, the cIEF process suffers from a major limitation that has prevented the direct examination of such complex mixtures derived from physiologic fluids. The presence of high salt concentrations (100-200 mM) disrupts the analyte focusing by perturbing or destroying the stationary $\mathrm{pH}$ gradient. Even low concentrations of salt (10-20 mM) can cause significant Joule heating that disrupts the formation of the analyte bands due to increased compound diffusion [6,9]. The salts increase the ionic strength and therefore the conductivity of the solution inside the capillary [9]. Higher currents are generated for a given voltage, thereby increasing the Joule heating effect. This can be overcome by use of a lower applied voltage, but this approach significantly reduces cIEF resolution. In order to alleviate this problem, the sample is typically desalted off-line prior to carrying out cIEF. Unfortunately, this leads to increased sample handling, resulting in significant sample losses. Recently, Wu and Pawliszyn [10] reported a desalting method using sample dialysis to remove salt and add 


\section{Al}
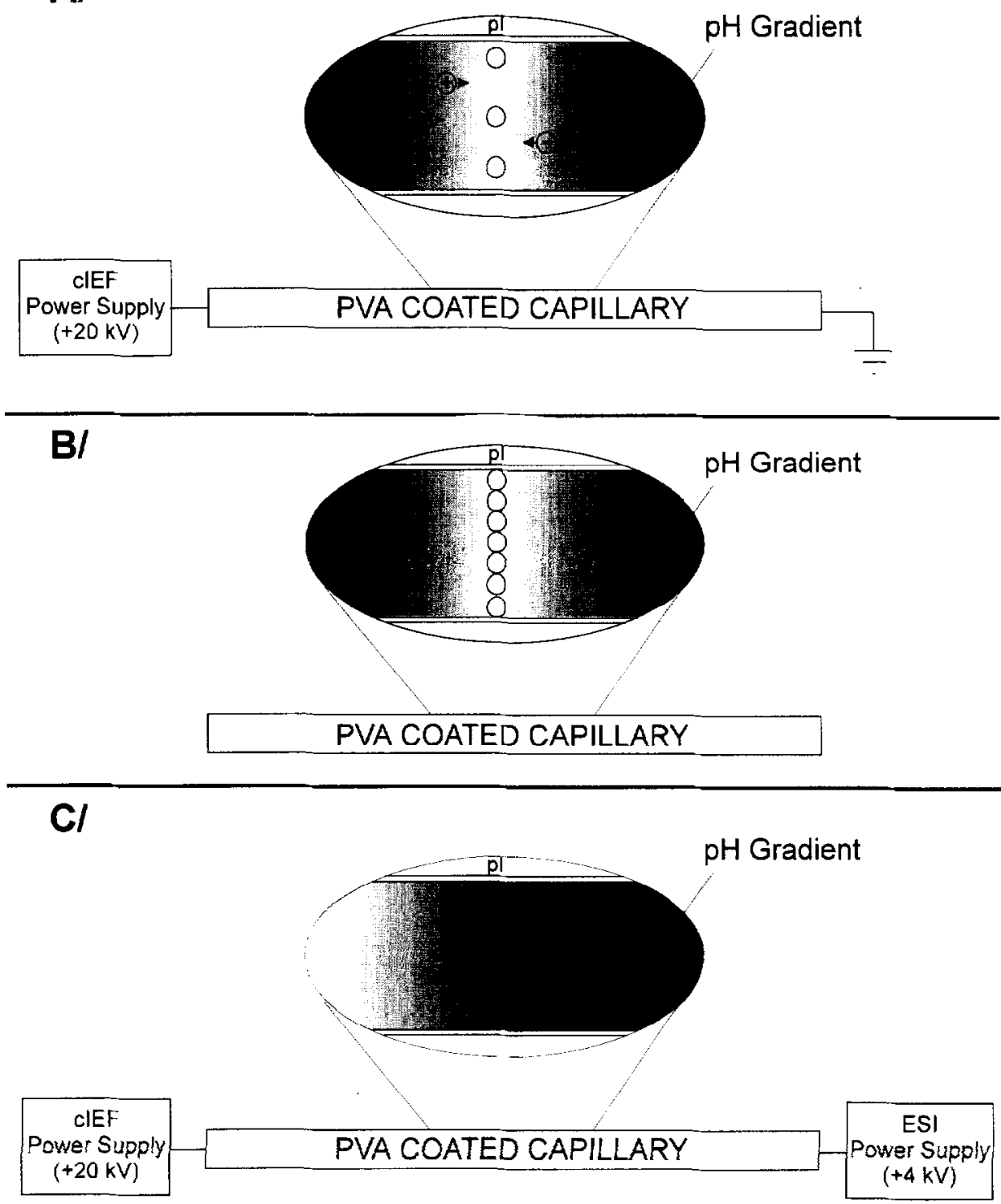

Figure 1. Schematic of the cIEF process within the capillary. (a) Charged analytes are focused within a stationary $\mathrm{pH}$ gradient provided by the ampholytes within the sample. (b) Upon reaching the $\mathrm{pH}$ value equal to their intrinsic pl value, the analytes become neutral and focused. (c) Electrophoretic mobilization occurs by replacing the catholyte with anolyte solution, allowing the neutral analytes to become protonated and migrate into the mass spectrometer.

the ampholyte. However, this elegant approach requires customized equipment and still occurs external to the separation capillary, leading to sample loss and reducing the potential to automate analyses.

We describe an on-line desalting method that requires no sample handling since we actually modify the standard cIEF procedure. This simple method takes place within the cIEF capillary that has been coated to reduce or negate the EOF and utilizes the much greater relative mobility of the salt anions and cations to allow their efficient removal. During this process there is minimum perturbation of the remaining analytes. In this work we demonstrate the usefulness of employing such on-line desalting of physiologically derived fluids in a one step analysis of CSF using cIEF-MS.

\section{Experimental}

\section{Materials}

Fused silica capillaries, $50 \mu \mathrm{m}$ i.d. $\times 360 \mu \mathrm{m}$ o.d. (Polymicro Technologies, Phoenix, AZ), were internally coated with polyvinylalcohol (PVA) (Aldrich Chemical Co., Milwaukee, WI) to reduce or eliminate the electroosmotic flow. Bovine heart cytochrome C (pI 8.6, 12360 Da) equine skeletal muscle myoglobin (pI 7.0, 16951 $\mathrm{Da}$ ), bovine erythrocyte carbonic anhydrase (pI 5.9, $29023 \mathrm{Da}$ ), and soybean trypsin inhibitor (pI 4.5, 23037 Da) were obtained from Calbiochem (La Jolla, CA); chicken egg lysozyme (pI 8.9, $14305 \mathrm{Da}$ ) and servalytes ( $\mathrm{pH}$ 3-10) were purchased from Sigma Chemical Co. 
(St. Louis, MO). Isolytes (pH 3-10) were obtained from ICN (Costa Mesa, CA). Cerebrospinal fluids were a gift from Dr. Noberto Guzman (RWJ Pharmaceutical Research Institute, Raritan, NJ) and Dr. Luis Hernandez (School of Medicine, Los Andes University, Merida, Venezuela). All reagents were analytical grade or better.

\section{Desalting}

The desalting protocol developed relies on the salt anions and cations being much smaller and more mobile than the analytes or ampholytes. By the application of a slow linear voltage ramp prior to focusing, it is possible to electrophoretically remove the majority of salt ions without disturbing the analytes or ampholytes. Once this is achieved, a conventional cIEF experiment is completed. The final voltage, voltage gradient, and ramp time required to properly desalt a solution are all a function of the salt concentration within the sample. A steep gradient or high final voltage will reduce the analysis time, but the likelihood of system perturbation is increased. Therefore, a balance between experimental run time and system perturbation was considered during method development. Typically, a linear voltage gradient from $0-10 \mathrm{kV}$ was applied over $6 \mathrm{~min}$. Great care was taken not to allow the current to reach higher than $15 \mu \mathrm{A}$, since in our experience greater values tended to degrade analyte resolution. The current would rise from $0-\sim 14 \mu \mathrm{A}$ in $\sim 3.5-4 \mathrm{~min}$, then plummet precipitously. This was the indication that the salt had migrated out of the cIEF capillary.

\section{cIEF-MS}

The separation capillary used in these experiments was coated internally with PVA utilizing a previously described method [11]. The dimensions of the separation capillary were $50 \mu \mathrm{m}$ i.d. $\times 360 \mu \mathrm{m}$ o.d. by $37 \mathrm{~cm}$ in length. Capillary IEF-MS analyses were performed using an in-house built cIEF interface coupled to a Finnigan MAT 900 sector mass spectrometer of EB geometry (where $E$ is the electrostatic analyzer and $B$ is the magnet). Analyte detection was by positive ion electrospray ionization mode at an operating resolution of 1000. A PATRIC ${ }^{\mathrm{TM}}$ (Position And Time Resolved Ion Counter) focal plane detector was used with an $8 \%$ mass window. The microchannel plate voltage was operated at a reduced voltage of $820 \mathrm{~V}$, which is optimal for the detection of multiply charged ions over singly charged species. The atmospheric pressure electrospray source was separated from the mass spectrometer vacuum by a heated metal capillary $\left(210^{\circ} \mathrm{C}\right)$. The separation capillary was passed through the inner bore of the electrospray needle and a sheath liquid junction formed the electrical contact between the voltage applied to the electrospray needle and the sample within the capillary. The cIEF apparatus consisted of a BA Model 205A-30P high voltage (HV) power supply (Bertan Associates, Syosset, NY).
Capillary IEF-MS experiments were completed in two stages. During focusing the separation capillary, filled with sample and ampholyte solution (1:1 mix of isolyte and servolyte), was withdrawn into the electrospray needle to afford a final ampholyte concentration of $0.5 \% \mathrm{v} / \mathrm{v}$. Catholyte (50\% methanol $/ 49 \%$ water $/ 1 \%$ ammonium hydroxide) was delivered coaxially to the capillary at a rate of $1 \mu \mathrm{L} / \mathrm{min}$. Sheath gas (nitrogen) was used at a flow rate $(0.28 \mathrm{~L} / \mathrm{min})$ that enabled the formation of a static "hanging" drop of catholyte at the tip of the spray needle [12]. The anolyte consisted of a solution containing $50 \%$ methanol $/ 49 \%$ water $/ 1 \%$ acetic acid $(v / v / v)$. The electrical circuit for focusing was completed by application of high voltage $(20 \mathrm{kV})$ to the anolyte while attaching an instrument ground wire to the ESI spray assembly. This voltage was applied across the capillary for $7 \mathrm{~min}$ and the current drawn from the high voltage supply followed through the mass spectrometer data system. Focusing was determined to be complete when the electrical current drawn from the high voltage supply had fallen below $10 \%$ of the initial current value.

Once focusing was complete, the electrical potential was turned off and the sheath liquid line was flushed with anolyte solution. The capillary tip was then passed out of the spray needle to a distance of $0.5 \mathrm{~mm}$. During analyte mobilization, anolyte solution was coaxially delivered as the sheath liquid, at a flow rate of $3 \mu \mathrm{L}$ per min. A constant voltage of $20 \mathrm{kV}$ was used to electrophoretically mobilize the focused anolyte bands through the capillary and into the electrospray source. A voltage of approximately $4 \mathrm{kV}$ with reference to the accelerating voltage $(5 \mathrm{kV})$ of the mass spectrometer was used throughout. The mass spectrometer was operated at a scan speed of $2 \mathrm{~s} /$ decade over a mass range of $1000-2500 \mathrm{~m} / \mathrm{z}$ units.

\section{Results and Discussion}

Although cIEF-UV has been widely utilized and characterized over the past decade, the limited analyte information obtained with UV detection has reduced the technique's utility for de novo research of in vivo derived samples. Capillary IEF-MS provides the enhanced analyte structural information required for such investigations, but has received scant attention $[7,8]$. To our knowledge, we are the first to report the use of cIEF-MS in the analysis of physiologic fluids.

A major limitation in the use of CIEF with physiologically derived samples has been the need to desalt them prior to analysis. Typically, this takes place off-line, which leads to increased experimental complexity and the chance of sample loss or contamination. In this study, we demonstrate a new on-line desalting technique that allows the direct analysis of in vivo derived samples. The technique relies on the large difference in mobility of small cations and anions when compared with polypeptides and proteins. A linear voltage gradient is applied across the separation capillary containing 


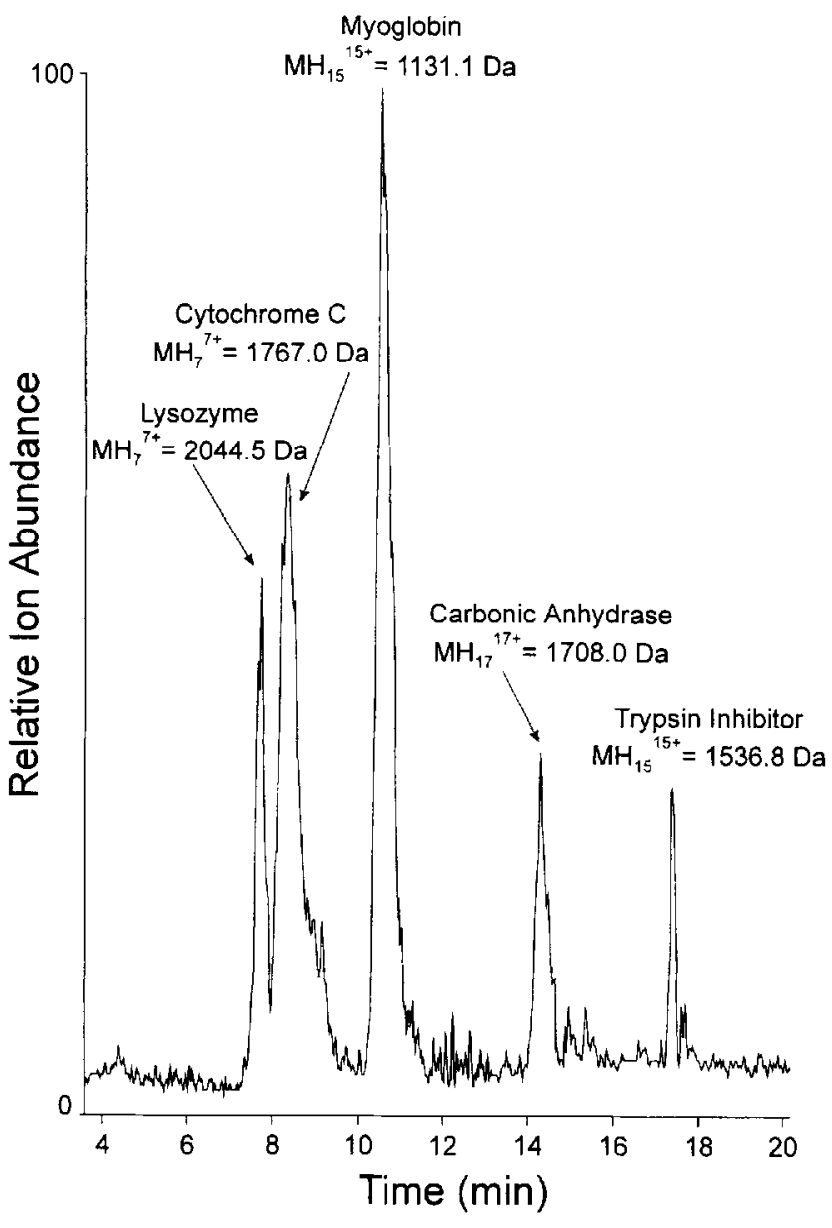

Figure 2. CIEF-MS single ion electropherograms recorded during the mobilization of the five protein mix doped with $200 \mathrm{mM}$ sodium chloride (focusing not shown). Focusing was achieved at a steady voltage of $10 \mathrm{kV}$ for $5 \mathrm{~min}$, followed by mobilization also at a steady voltage of $10 \mathrm{kV}$. Detection was by PATRIC ion counter operated at $820 \mathrm{~V} /$ microchannel and a mass window of $8 \%$. The mass spectrometer was operated over a mass range of 1000-2500 $\mathrm{Da}$ at a scan speed of $2 \mathrm{~s} /$ decade.

the sample mixed with ampholytes. This gradient is constructed to remove the swiftly migrating small molecules while causing the minimum of perturbations to the forming $\mathrm{pH}$ gradient. The passage of ions off the capillary can be followed by an examination of the current drawn from the CIEF power source. A sharp drop in current from $\sim 14$ to $\sim 1.5 \mu \mathrm{A}$ indicates the removal of the majority of salt and other small molecules from the CIEF capillary and demonstrates that the system is ready for the IEF process.

In order to determine the efficacy of the desalting method, a mixture of five commercially available proteins in aqueous solution containing $200 \mathrm{mM} \mathrm{NaCl}$ was analyzed by cIEF-MS. The protein mixture consisted of 500 $\mathrm{ng} / \mu \mathrm{L}$ of each protein, comprising lysozyme, cytochrome C, myoglobin, carbonic anhydrase, and trypsin inhibitor. The ion electropherogram derived from monitoring the most abundant charge state of each protein is shown in Figure 2. The migration times, ion intensities, and resolu-

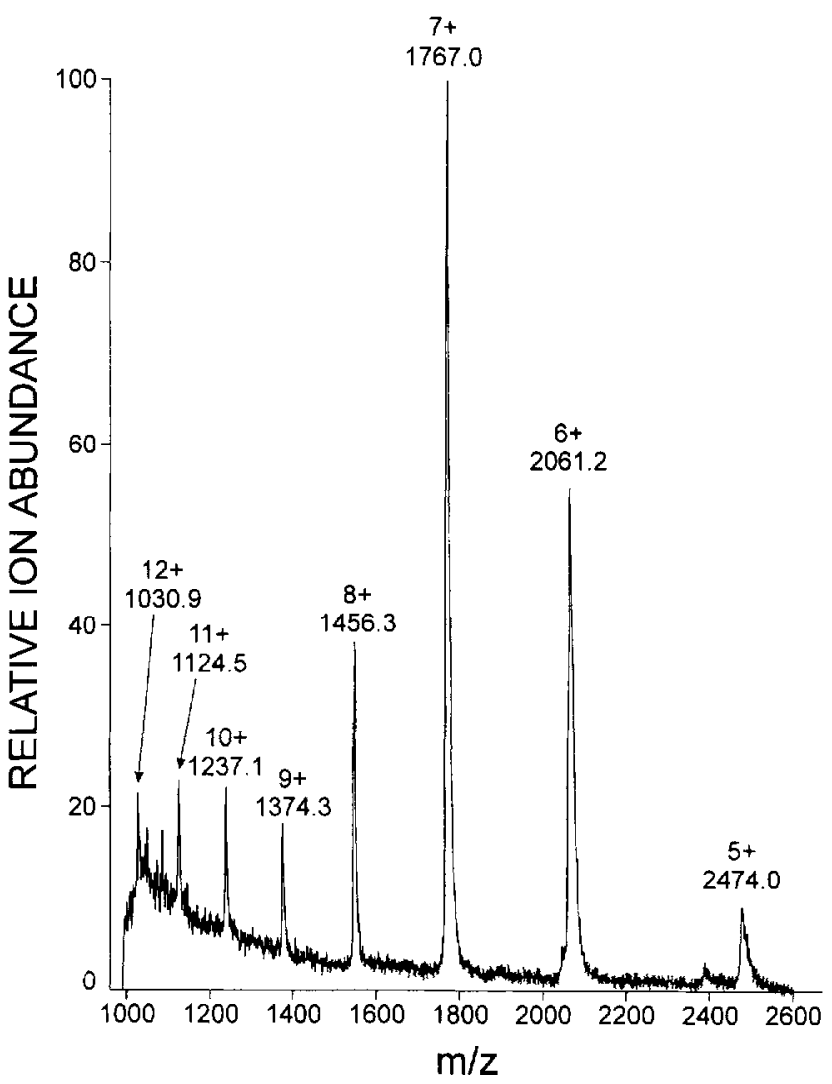

Figure 3. CIEF-MS multiply charged ion spectrum of cytochrome C. Conditions as described in Figure 2. Ampholyte signals appear as singly charged ion species in the mass range $\sim 950-1100$.

tion were comparable to previous experimental data obtained from analysis of the mixture without $\mathrm{NaCl}$ added (data not shown). The analyte migration order was as expected according to the literature $\mathrm{pI}$ values. However, it should be noted that after mobilization of the analytes, it is not possible to determine directly the exact $\mathrm{pl}$ value of each protein. This is due to the fact that mobilization results in a nonlinear $\mathrm{pH}$ gradient; hence, migration times cannot be used to estimate $\mathrm{pI}$ values [10].

The desalting step readily removes interfering cations and anions from solution prior to spraying into the ESI source. However, the remaining presence of ampholyte in the cIEF capillary does not significantly impact on ESI-MS performance [7,8,12]. Although there is some reduction in ionization efficiency of the analyte proteins, this is compensated by the fact that the clEF process is a concentration, as well as a separation process [7]. Another factor to consider is that the concentration of individual components in the ampholyte mixture is exceedingly low and the overall concentration of the mixture is $0.5 \% \mathrm{v} / \mathrm{v}$. Furthermore, the mass range of the singly charged ampholytes is $\sim 950-1100$, outside the mass range of most multiply charged ions originating from protein analysis. A typical multiply charged ESI-MS spectrum of a protein by cIEF-MS after desalting is shown in Figure 3. The signal:chemical noise (ampholyte) for the most abun- 
a.

b.
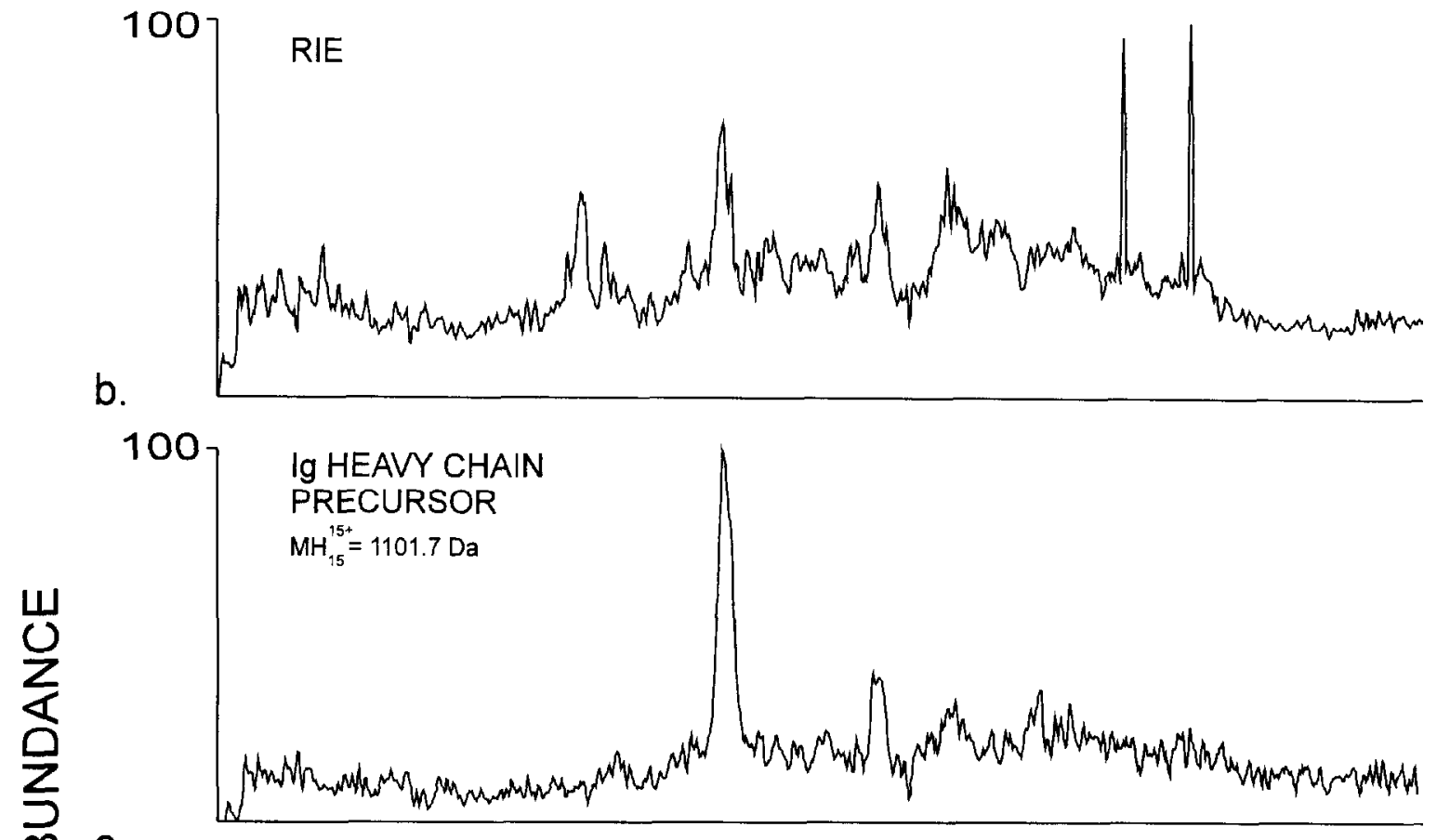

C.

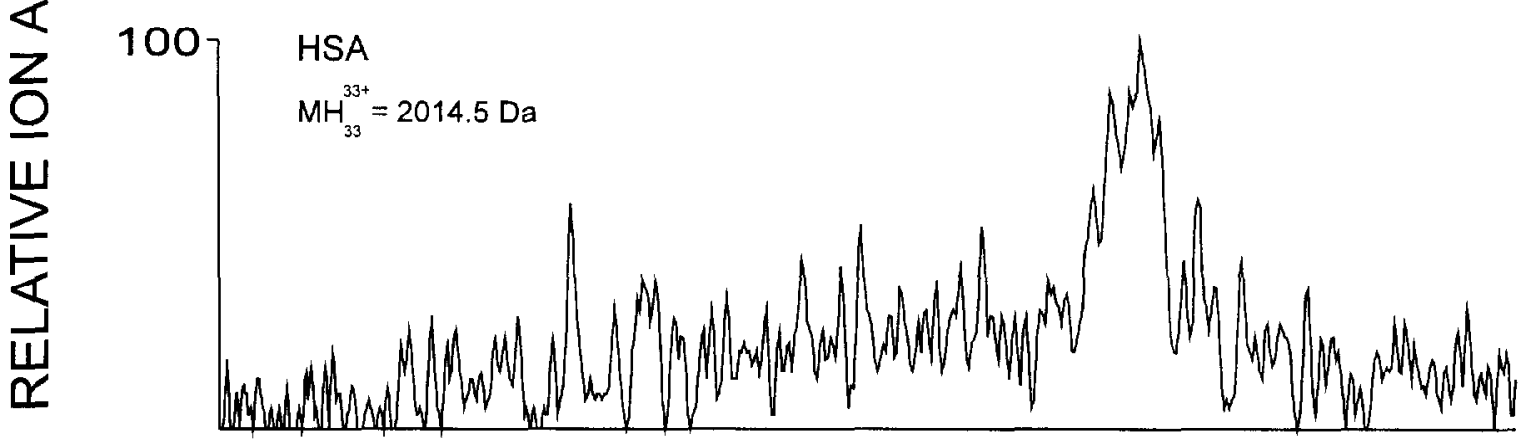

d.

100

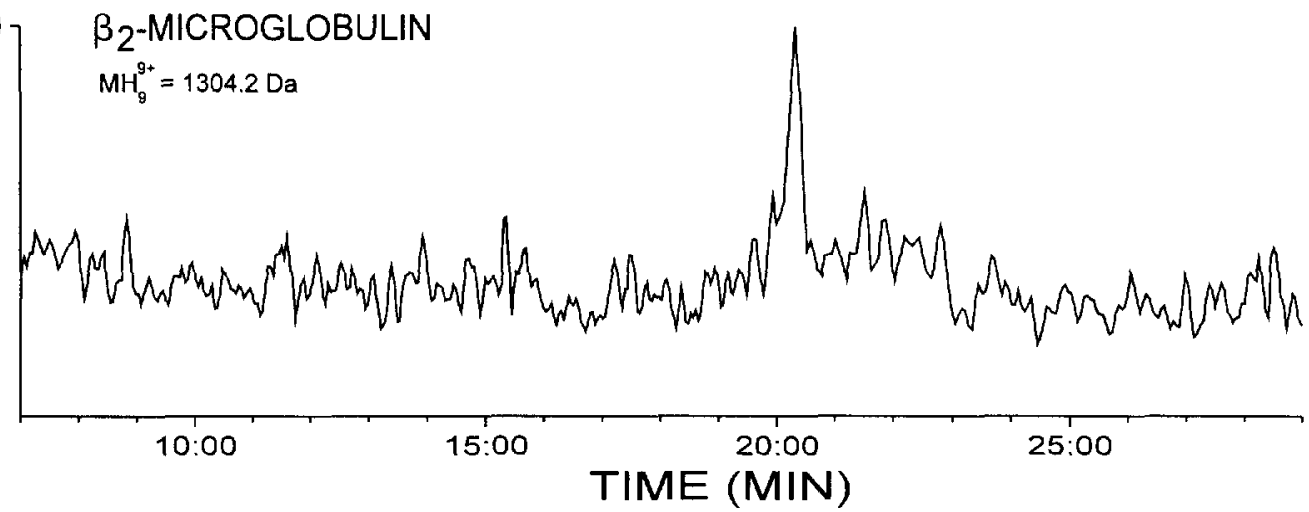

Figure 4. CIEF-MS data from examination of human cerebrospinal fluid (CSF). (a) Reconstructed ion electropherogram demonstrating the complexity of this physiological sample. Many andytes have been clearly resolved from this sample. (b) Tentative identification of $\mathrm{Ig}$ heavy chain precursor. (c) Identification of human serum albumin (HSA). (d) Identification of $\beta_{2}$-microglobulin within the CSF. These analytes appear as some of the most intense within the data. All analytes were identified by molecular weight data. 
dant multiply charged ions is $\sim 50: 1$ for the analysis of cytochrome $\mathrm{C}$. There is some slight overlap of ampholyte signal in the mass range $-1000-1100 \mathrm{Da}$, but the signal response for the proteins is clearly discernible above background.

The results on the standard protein mixture proved that the desalting method is highly effective, while only increasing run time by $4-6 \mathrm{~min}$. As sample desalting occurred within the capillary, minimal sample handling, and hence minimal analyte losses, occurred, thereby affording a method for analysis of physiological fluids. In order to show the usefulness of this approach, we directly analyzed human cerebrospinal fluid (CSF). This physiological fluid consists of a highly complex matrix of salts and small molecules. Initially we attempted to directly analyze by cIEF-MS the complex protein mixture present in CSF with no prior sample preparation and no on-line desalting. This resulted in very high capillary currents $(>40 \mu \mathrm{A})$ and intense Joule heating. Furthermore, no analyte signals were detected at the mass spectrometer due to electrospray ionization instability caused by the presence of high salt concentrations. Subsequently, $1 \mu \mathrm{L}$ of CSF was mixed directly with ampholytes with no other sample preparation and pressure injected onto the cIEF capillary. The desalting voltage ramp was applied as described previously, followed by cIEF-MS analysis. The reconstructed ion electropherogram (RIE) obtained was exceedingly complex and revealed the presence of a large number of resolved analytes (Figure 4). The RIE was made more complex by the presence of ampholyte responses. However, a tentative identification of oxidized Ig heavy chain precursor could be made (Figure $4 \mathrm{~b}$ ). This assignment was tentative due to the presence of only three readily identifiable charge states for this protein $\left(16^{+}\right.$ charge state, $\mathrm{m} / z=1032.8 \mathrm{Da}, 100 \%$ relative abundance; $15+$ charge state, $m / z=1101.7 \mathrm{Da}, 85 \%$ relative abundance; $14+$ charge state, $m / z=1180.3 \mathrm{Da}, 35 \%$ relative abundance). However, it was possible to identify other compounds present in the CSF whose charge state envelopes fell at higher $m / z(>1300 \mathrm{Da})$ values and away from the main ampholyte signals $(<1100 \mathrm{Da})$. For example, a response was detected for human serum albumin (HSA), a major component of CSF $\left(m / z\right.$ 2014.5, $M_{r}=$ $66430 \mathrm{Da}$ ) (Figure 4c), as well as an abundant response at $\mathrm{m} / z$ 1304.2 Da corresponding to the $9^{+}$charge state of $\beta_{2}$-microglobulin $\left(M_{r}=11729 \mathrm{Da}\right)$ (Figure $\left.4 \mathrm{~d}\right)$. Overall, these analyses proved the viability of the desalting technique while highlighting the ability of cIEF-MS to provide high resolution separation and swift identification of analytes within physiological fluids.

\section{Conclusions}

This study has shown cIEF-MS to be a powerful analytical technique for use with in vivo derived fluids. The technique is hampered, however, by the need to desalt the sample prior to analysis. The desalting protocol developed during this investigation overcomes this by allowing on-line desalting as part of an automated experiment. Furthermore, this desalting occurs within the same capillary in which cIEF separation subsequently takes place, thereby reducing sample loss or contamination to a minimum. When used in conjunction with cIEF-MS, this desalting protocol allows swift and sensitive analyses of complex physiological fluids such as human CSF. The utility of this approach is that complex protein mixtures derived from biological fluids and cells can be readily analyzed in a single step analysis. This alleviates any losses due to extensive sample handling and allows rapid determination of the presence of proteins in complex mixtures.

\section{References}

1. Hjertén, S.; Zhu, M.-D. J. Chromatogr. 1985, 346, 255-270.

2. Liu, X.; Sosic, Z.; Krull, I.S. J. Chromatogr. A 1996, 735, 165-190.

3. Pritchett, T.J. Electrophoresis 1996, 17, 1195-1201.

4. Wu, J.; Pawliszyn, J. J. Chromatogr. B 1994, 657, 327-332.

5. Nelson, J.R.; Burgi, D.S. Handbook of Capillary Electrophoresis; Landers, J.P., Ed.; CRC: Boca Raton, FL, 1994; p 549.

6. Zhu, M.; Rodriguez R.; Wehr, T. I. Chromatogr. 1991, 559, 479-488.

7. Tang, Q.; Harrata; A.K.; Lee, C.S. Anal. Chem. 1995, 67, 3515-3519.

8. Tang, Q; Harrata, A.K.; Lee, C.S. Anal. Chem. 1996, 68, 2482-2487.

9. Grushka, E.; McCormick, R.M.; Kirkland, J.J. Anal. Chem. 1989, 61, 472-479.

10. Wu, J.; Pawliszyn, J. Anal. Chem. 1995, 67, 2010-2014.

11. Clarke, N.J.; Tomlinson, A.J.; G. Schomburg; Naylor, S. Anal. Chem., in press.

12. Kirby, D.P., Thorne, J.M., Götzinger, W.K., Karger, B.L. Anal. Chem. 1996, 68, 4451-4457. 